Voix et Images

\title{
Une ou des littératures canadiennes? une entrevue avec D.G. Jones
}

\section{Richard Giguère}

Volume 10, numéro 1, automne 1984

Littérature canadienne-anglaise

URI : https://id.erudit.org/iderudit/200454ar

DOI : https://doi.org/10.7202/200454ar

Aller au sommaire du numéro

Éditeur(s)

Université du Québec à Montréal

ISSN

0318-9201 (imprimé)

1705-933X (numérique)

Découvrir la revue

Citer ce document

Giguère, R. (1984). Une ou des littératures canadiennes? une entrevue avec D.G. Jones. Voix et Images, 10(1), 5-22. https://doi.org/10.7202/200454ar d'utilisation que vous pouvez consulter en ligne.

https://apropos.erudit.org/fr/usagers/politique-dutilisation/ 


\section{Une ou des littératures canadiennes? une entrevue avec D.G. Jones}

par Richard Giguère, Unịversité de Sherbrooke

Poète, critique, traducteur, Douglas Gordon Jones enseigne au Département d'anglais de l'Université de Sherbrooke depuis plus de vingt ans. Ses recueils publiés sont Frost on the Sun (1957), The Sun Is Axeman (1961), Phrases from Orpheus (1967); Under the Thunder the Flowers Light Up the Earth (prix du Gouverneur général, 1977) et $A$ Throw of Particles: the New and Selected Poetry of D.G. Jones (1983). Son livre de critique paru en 1970, Butterfly on Rock: a Study of Themes and Images in Canadian Literature, a connu trois rééditions et est devenu un classique de la critique littéraịre canadienne. Il a de plus publié des dizaines d'articles dans des revues canadiennes et américaines comme Canadian Literature, Queen's Quarterly, Boundary 2, Credences, Ellipse, Canadian Poetry, etc. Jones est surtout connu des milieux littéraires francophones du Québec comme traducteur de poésie. Depuis qu'il a fondé la revue Ellipse en 1969, il a traduit de nombreux textes de poètes québécois, surtout ceux de sa génération: Fernand Ouellette, Gaston Miron, Roland Giguère, Jacques Brault, Gilles Hénauilt, Michèle (Lalonde, mais aussi Alain Grandbois, Rina Lasnier, Alfred DesRochers parmi. les aînés et Michel Garneau, Paul Chamberland, Nicole Brossard parmi les jeunes. L'International Poetry Forum et les Presses de l'Université. de Pittsburg ont publié un choix de poèmes de Paul-Marie Lapointe traduit par lui en 1976, The Terror of the Snows.

J'ai rencontré Doug Jones chez lui, à North Hatley, en avril 1984. $L$ 'entrevue a eu lieu en anglais, et $j$ 'ai moi-même adapté et transcrit en francais ses propos. Je remercie Monique Grandmangin qui a patiemment relu et annoté le manuscrit de l'entrevue avant sa dactylographie finale. 


\section{Le mythe fondateur de la littérature canadienne}

\section{V.I. Quand naît la littérature canadienne? D'où vient-elle?}

D.J.Elle naît à peu près en même temps que la littérature québécoise, vers 1820. Il s'écrit alors toutes sortes de poèmes, surtout des longs textes que j'appellerais des poèmes topographiques qui décrivent la nature, les paysages, l'environnement géographique. Il est intéressant de voir les affinités de cette poésie avec la peinture réaliste pratiquée alors par des officiers de l'armée ou leurs épouses. Cette peinture est enseignée dans les écoles militaires, elle fait partie d'une tradition académique qui vient d'Angleterre. La poésie canadienne à ses débuts reprend cette manière réaliste. On voit tout de suite la différence avec la peinture québécoise de la même époque, qui reproduit essentiellement un paysage symbolique, car il s'agit d'une peinture religieuse. Ou alors il y a la tradition picturale des portraits. Il n'y a donc pas au Québec à cette époque une peinture réaliste, une peinture de paysage comme au Canada. On peut noter la même différence entre la littérature canadienne et la littérature québécoise, en particulier en ce qui a trait à la poésie. Les préoccupations reliées à l'espace, aux lieux géographiques concrets sont fondamentales chez les poètes canadiens alors qu'on ne peut pas dire la même chose de la poésie québécoise. Bien sûr il y a des poèmes qui tiennent compte de la nature, du paysage québécois, mais la vision n'est pas la même.

V.I. Ainsi, la littérature, la poésie canadienne se youlaient réalistes dès le départ. Pourtant je croyais que l'influence romantique sur la littérature du dix-neuvième siècle était marquante, non?

D.J.Ả mon avis les textes les plus importants de cette époque sont les longs poèmes écrits depuis le début du siècle jusque vers $1880-1890$, et cela d'un bout à l'autre du pays : ceux d'Oliver Goldsmith à Halifax, comme "The Rising Village», de William Kirby ou d'Alexander McLachlan du Haut Canada, d'Isabella Valancy Crawford entre autres. Tous ces longs poèmes racontent la même histoire, même si le style varie de l'un à l'autre. Il s'agit du pionnier qui transforme la nature sauvage en une nature domestiquée, c'est-à-dire qui repousse-la forêt et défriche son lopin de terre, construit sa maison, trace des routes et va même jusqu'à créer des petites villes. C'est là la thématique centrale d'une bonne demi-douzaine de longs poèmes. Voilà donc le récit, le mythe fondateur de la littérature canadienne, qui consiste à instituer dans ces terres incultes un ordre social.

Le débat qui prend forme dans ces longs poèmes concerne le type de nature domestiquée qui résultera de l'action du pionnier et le rapport du pionnier avec son nouvel environnement. L'alternative est la suivante: fonder, mettre sur pied un empire, avec le type de rapports 
que cela implique, ou alors privilégier une relation personnelle et intime avec la nature. Â ce sujet il y a une ambiguité qui se fait jour dès le départ, car le pionnier voudrait à la fois procéder à la fondation de l'empire et conserver sa relation intime avec la nature. Mais plus le temps passe, plus la fin du dix-neuvième siècle approche, plus on se rend compte que les deux visions sont incompatibles. L'une sousentend l'idée de conquête de la nature et d'expansion territoriale, c'està-dire l'exploitation des ressources (forêts, mines, etc.) et les grands projets qui en découlent, comme la construction d'un chemin de fer. Cețte vișion entraîne un rapport impersonnel avec l'environnement et se définit surtout comme un rapport d'exploitation, un viol de la nature. 'L'autre, la vision intimiste, qui se traduit par un réseau de métaphores pastorales, suppose plutôt un respect de la nature et un système de valeurs correspondant. Dans les longs poèmes, cette vision est symbolisée par le rapport du pionnier avec son épouse ou une femme. L'épouse et le foyer constituent l'axe privilégié autour duquel s'organise le poème et il s'agit alors d'une relation amoureuse, érotique. À la place du viol qui découle de la vision impériale, la vision intimiste conduit au mariage. On voit donc que la thématique originale de la poésie canadienne, fondée sur ce conflit entre deux visions et deux systèmes de valeurs, est très différente de la thématique de la poésie québécoise.

V.I. Mais que devient l'imaginaire dans ces textes? En poésie québécoise, même si Fréchette écrit sur le Mississipi, même si Crémazie dans "Chant du vieux soldat canadien» se souvient avec nostalgie de la France, l'imaginaire occupe une place importante. Et je pense au cas de Nelligan qui est encore plus probant. Est-ce qu'il n'y a, au dixneuvième siècle, qu'une poésie réaliste au Canada anglais?

D.J.Il n'y a pas que des paysages vus de façon réaliste, même s'il faut bien admettre que c'est surtout de cela dont il est question. La problématique centrale de la littérature et de la culture canadiennes, je l'ai dit, concerne le rapport que l'homme entretient avec le monde. Cela peut vouloir dire l'environnement géographique, comme les champs, la ferme, la forêt, mais aussi les rapports avec les autres hommes, les voisins, la femme aimée. C'est ici que l'imaginaire intervient. McLachlan par exemple affirme qu'il y a une relation mystérieure entre les hommes et les choses matérielles, ce qui suppose une communion avec des forces obscures, une sorte de présence divine anonyme. On peut dès lors parler d'un rapport religieux ou spirituel avec ces forces obscures, mais médiatisé par le monde extérieur.

Ce qui se produit en poésie québécoise, c'est un rapport avec un idéal religieux, métaphysique, abstrait dont le langage est l'intermédiaire. Il y a bien sûr des symboles comme le drapeau de Carillon chez Crémazie, ou des objets d'art, mais le langage demeure l'intermédiaire essentiel. La littérature québécoise a été pendant cent cinquante ans surtout verticale, à la recherche d'un idéal souvent absent. Que cet idéal ait comme 
objet l'histoire, la nostalgie d'une Nouvelle-France disparue pour Crémazie, ou l'art comme c'est le cas chez Nelligan, c'est-à-dire la quête de la pureté de l'art, de la poésie ou alors une pureté religieuse, morale, spirituelle associée à la femme, le problème est de savoir si on peut atteindre cet idéal. Et il semble qu'il soit de plus en plus difficile pour le poète de s'identifier à son idéal. Quand cela n'est plus possible, le monde devient absurde et vie. C'est ce qui arrive dans l'œeuvre de Crémazie, en partie aussi chez Nelligan et très certainement chez SaintDenys Garneau. Pour le vieux soldat de Crémazie, il n'y a plus que des champs de bataille gelés, vides. Le Québec ne représente plus à ses yeux, pour reprendre l'expression de Voltaire, que quelques arpents de neige. Chez Garneau le monde n'est plus qu'une sphère poussiéreuse, une figure géométrique vide. Le monde réel ne réside pas pour ces poètes dans leur environnement géographique et physique, mais dans un idéal impossible à atteindre.

V.I.C'est pour vous une différence fondamentale entre les deux littératures?

D.J.Oui. Pour les écrivains et les artistes canadiens, la vision est avant tout horizontale et liée à l'espace. Il n'y a qu'à lire les poètes du dixneuvième siècle et encore plus les poètes du vingtième siècle comme Edwin John Pratt, Earle Birney, Al Purdy ou Raymond Souster pour s'en convaincre. En fait on n'a qu'à relire les titres de leurs poèmes. Plusieurs de ces titres nous situent dans le temps et dans l'espace. Le titre d'un poème de Souster nous donne l'heure exacte, 2 heures de l'après-midi ou 2 heures de la nuit, et l'endroit précis, telle rue de Toronto, où se déroule l'action. Birney rapporte fidèlement dans son titre le lieu où se trouve le narrateur, quelque part en Amérique du Sud ou en Amérique centrale, et il décrit en détail ce lieu et dit l'heure à laquelle l'événement se produit. Dans les longs poèmes de Pratt, The Titanic (1935), The Roosevelt and The Antinoe (1930), Towards the Last Spike (1952) ou Dunkirk (1941), tout est rapporté à la minute et à la seconde près.

\section{La modernité en poésie}

V.I. En gros donc, la littérature canadienne du dix-neuvième siècle s'intéresse avant tout à la nature, à l'environnement et les décrit de façon réaliste. De son côté la littérature québécoise se préoccupe plus du temps, le passé par exemple, et est à la recherche d'un idéal. Elle fonctionne selon un axe vertical plutôt qu'horizontal. Mais alors, comment toute cette problématique évolue-t-elle au vingtième siècle? Comment naît ce qu'on appelle la modernité en poésie canadienne? Quand voiton apparaître une thématique moderne, celle de la ville par exemple? 
D.J.La ville est déjà un thème important au dix-neuvième siècle, peut-être pas dans le sens précis que nous donnons au mot aujourd'hui. La présence de la ville est symbolisée par la cabane du pionnier qui est une première marque de civilisation. Dans "The Rising Village» de Goldsmith par exemple, le petit village devient une ville. Et en général le poète affiche une attitude positive face à la nouvelle réalité urbaine. En fait la vision pastorale de Goldsmith est très positive: il célèbre l'intégration harmonieuse de la ville à l'environnement naturel de la campagne. Avec l'arrivée sur la scène littéraire, à la fin du siècle dernier, du groupe qu'on a appelé les poètes de la Confédération (Bliss Carman, Archibald Lampman, Charles G. D. Roberts, Duncan Campbell Scott), on assiste à la fin de cette intégration harmonieuse de la ville et de la campagne, de la culture et de la nature. Ces poètes sont plutôt pessimistes quant aux chances de succès du rêve d'intégration. Le poème "The City of the End of Things» de Lampman représente une vision diabolique de la ville. Il s'agit d'une ville de machines où tout est automatisé et déshumanisé. Sans doute une réussite technique de la société industrielle mais marquée en contrepartie par un vide spirituel total pour les citoyens. Lampman et les autres poètes du groupe rejettent la vision impérialiste dont $j$ 'ai parlé tantôt, l'exploitation impersonnelle des biens et de l'homme par l'homme. Ils tournent donc le dos à la ville et retournent à la campagne.

V.I. Tout cela est très romantique, non?

D.J.Bien sûr, Lampman par exemple est très influencé par Wordsworth et les romantiques anglais. Mais ces influences, une fois assimilées, s'intègrent bien à la vision des poètes de la Confédération. Nous assistons au déclin de l'image du héros-pionnier. L'homme des bois plus grand que nature, le bûcheron légendaire est devenu un bûcheron de fin de semaine, un modèle déclassé du héros original, qui coupe maintenant du bois pour quelques-uns de ses amis et de ses voisins, s'il le peut. Car ce nouveau héros est isolé, il a choisi de vivre seul, célibataire, afin de sauvegarder sa relation privilégiée avec la nature. Sa santé physique est excellente, tous ses sens sont aiguisés et il peut ainsi décrire en détail la faune et la flore, les saisons, l'automne en particulier. Mais tous ces textes sont empreints de mélancolie parce que l'homme, pour préserver son rapport personnel à la nature, doit s'éloigner de la communauté des autres hommes.

V.I. On comprend dès lors la violence de la confrontation qui met aux prises les poètes de la Confédération et les premiers poètes vraiment urbains. Je pense aux membres du groupe de McGill dans les années vingt, Arthur Smith, Frank Scott, Leo Kennedy qui changent non seulement le contenu de la poésie canadienne, mais aussi la forme, le langage poétique. Ce n'est pas à mots couverts que ces auteurs balaient du revers de la main ce que Kennedy appelle le «Maple Leaf School» en parlant des poètes de la Confédération. Il ne cesse de s'exclamer : "Entrons dans le vingtième siècle, et au plus vite!" 
D. J.Le groupe de McGill au complet, en collaboration avec Pratt de Toronto, lutte pour promouvoir une poésie résolument moderne au Canada. Scott et Smith veulent créer une poésie rigoureuse, intellectuellement stimulante et engagée dans les problèmes urbains. Smith, plus que Scott, est influencé par des modèles européens, les symbolistes français, William Butler Yeats, Thomas Stearns Eliot. Eh bien, malgré les points communs entre Scott et Smith, la poésie intellectuelle, la verve ironique et sarcastique, l'engagement social, Scott reprend les thèmes et une partie de la problématique des poètes du dix-neuvième siècle. Une des préoccupations majeures de sa poésie est celle-ci : comment réussir à bâtir une société dynamique, qui défende un système de valeurs opposé à l'exploitation capitaliste? Il faut rappeler que, dans sa vie comme dans son œuvre, l'idéal politique de Scott est la socialdémocratie telle que conçue par la CCF (Commonwealth Cooperative Federation), l'ancêtre du NPD, dans les années trente. Dans un poème comme "Laurentian Shield", alors que le narrateur se tourne vers le nord et regarde les champs, les chemins de fer, les routes, il est clair qu'il met en avant une vision socialiste de l'homme et de son environnement, une vision qui se démarque nettement de l'exploitation capitaliste. Le poème se termine par l'image d'une communauté qui, ayant défriché la terre et s'y étant installée à demeure, a réussi à transformer les pierres en petits enfants. Sous la plume de Scott, ce motif de l'intégration harmonieuse de l'homme à son environnement nous ramène à la problématique centrale de la littérature canadienne du dixneuvième siècle, aux deux visions du monde et aux deux systèmes de valeurs dont $j$ 'ai parlé tout à l'heure. Scott espère que le genre de socialisme qu'il prône réussira à réduire ou à contenir la poussée impérialiste.

V.I. Est-il le seul à défendre cette idéologie dans les années trente et quarante?

D.J.Pratt est un cas différent. C'est un auteur qui ne s'intéresse pas à la poésie symboliste, il s'intéresse à peine aux théories des imagistes américains. Il écrit plutôt des poèmes narratifs, traditionnels si on les compare aux courants modernes de poésie anglaise et française. Depuis Wordsworth, dont les longs poèmes traitent de lui-même, de son propre cheminement, on dit que la poésie moderne s'est tournée vers l'intérieur de l'homme, en mettant l'accent sur ses dimensions intellectuelle et spirituelle. Mais Pratt va à l'encontre de cette démarche. Il rédige des poèmes qui racontent des histoires héroïques ou épiques et qui à première vue semblent tout à fait traditionnels. Pourtant il est moderne dans le sens où les histoires qu'il raconte le sont aussi, comme le nauffrage du Titanic, la construction du chemin de fer du Canadien Pacifique ou l'évacuation de Dunkerque. De plus, Pratt est fasciné par les machines, que ce soit les bateaux à vapeur, les sous-marins, le nouvel 
équipement du Titanic, et par les découvertes récentes en géologie ou en chimie. Tout cela se retrouve en détail dans son œuvre. De ce point de vue sa poésie est très représentative des changements techniques et scientifiques de la première moitié du vingtième siècle, beaucoup plus représentative en tout cas que l'oeuvre de nombreux poètes qui ont choisi de ne pas parler de ces changements pour concentrer leur attention sur l'homme intérieur. En somme Pratt est directement branché sur les grandes réalisations modernes, sur l'ère des machines et de la technologie. Ses poèmes sont extrêmement bien documentés (dans le sens que Dorothy Livesay donne au mot «documentaire»).

\section{Une ou des littératures canadiennes?}

V.I. Le roman et la poésie des années trente et quarante sont de plus en plus l'affaire des villes, Montréal et Toronto d'abord, puis Vancouver plus tard dans les années soixante. Peut-on parler d'une mosaïque de la littérature canadienne, avec différents centres régionaux, un peu comme on a parlé d'une mosaïque de la société canadienne? Y a-t-il une ou des littératures canadiennes? On parle de plus en plus d'une littérature de la côte Pacifique, d'un roman de la Prairie, d'une poésie québécoise de langue anglaise, d'une littérature des provinces maritimes.

D.J.Le régionalisme est un sujet à la mode en littérature canadienne depuis quelques années. Certains auteurs des provinces de l'Ouest par exemple affirment: nous avons été colonisés par l'Est pendant des décennies, le temps est venu de découvrir notre propre identité, d'écrire notre roman et notre poésie à nous. La spécificité régionale s'appuie d'abord sur la réalité du Canada qui est un pays immense. Mais personnellement je doute de la pertinence de cette spécificité. Du dix-neuvième siècle jusqu'à nos jours, jusqu'à Robert Kroetsch en fait, il n'y a, à mon avis, qu'une seule littérature canadienne-anglaise. Cela n'empêche pas de constater l'existence de certaines particularités régionales lorsqu'on se déplace des provinces maritimes à l'Ontario urbain ou rural et jusqu'à la côte Pacifique en passant par la Prairie. En poésie on compte quelques mouvements littéraires excentriques par rapport à l'Ontario ou au Québec: La revue Tish (1961-1969) et l'influence des poètes de «Black Mountain " (William Carlos Williams, Charles Olson, Robert Creely et d'autres) à Vancouver dans les années soixante en sont un bon exemple. Et pourtant, même dans ce cas, ce mouvement se fusionne avec le groupe de la revue Open Letter (depuis 1965) à Toronto dans les années soixante-dix et poursuit ses contacts avec certains poètes américains. On oublie que de pareils contacts avaient déjà été établis par Dudek et Souster dès les années quarante et cinquante.

V.I. Mais ces deux revues bouleversent passablement la scène littéraire de l'époque, vous ne croyez pas? 
D.J.Tish et Open Letter n'apportent rien de vraiment révolutionnaire à la poésie canadienne des années soixante. L'accent mis par les poètes de «Black Mountain» sur l'image, sur l'espace immédiat de' la communauté, sur le langage particulier de ce lieu, sur le corps et la respiration, l'acte physique d'écrire chez Charles Olson, tout cela sur quoi Tish et Open Letter mettent l'accent à leur tour ne fait que reprendre des idées, des concepts, des tendances qui étaient déjà là en poésie canadienne-anglaise. Le mouvement de Tish vient donc renforcer ces préoccupations, il ne change pas la direction de la poésie. Il y a par contre des poètes qui ont voulu réorienter la poésie canadienne de façon plus radicale et qui ont été influencés dans leur démarche par Northrop Frye. On les a appelés les «myth poets », ce sont par exemple Jay Macpherson, Gwendolyn MacEwen et James Reaney. $\grave{A}$ mon avis ces poètes intéressés par le symbolisme et les mythes tentent vraiment de donner une nouvelle direction à la poésie canadienne qui présente des affinités avec la poésie québécoise: On sent très bien qu'ils sont en marge du mouvement général de la poésie canadienne. D'Alden Nowlan, le poète du Nouveau-Brunswick qui écrivait des poèmes marqués de l'empreinte de William Carlos Williams, à Louis Dudek de Montréal et Raymond Souster de Toronto, tous deux se situant dans le sillage des Américains Ezra Pound, Charles Olson et Robert Creely, jusqu'à George Bowering et ses amis de Vancouver, la poésie canadienne se trouve soit du côté du réalisme social, du poème «documentaire» (Dorothy Livesay), soit du côté de la poésie moderne conçue à la façon des poètes de «Black Mountain».

V.I. Vous venez de parler de mouvements poétiques et de poètes de différentes régions du Canada, à l'exception de la Prairie. Est-ce que cette région est différente des autres?

D.J.Non, le parti pris réaliste se retrouve partout au pays, du dix-neuvième siècle jusqu'à aujourd'hui. Prenons l'exemple d'un romancier de la Prairie, Robert Kroetsch, qui écrit maintenant de la poésie. Il a publié une série de poèmes qu'il a rassemblés dans Field Notes (1981). Dans certaines suites de ce livre comme «Seed Catalogue» (1977) et «The Ledger » (1975), Kroetsch tente de retrouver ses racines dans les Prairies et de définir l'Ouest canadien en termes précis. Mais il s'aperçoit bientôt que ce retour aux sources le ramène en Ontario, dans le contexte des pionniers du dix-neuvième siècle, que j'ai évoqué tout à l'heure. Mine de rien, ces textes se rattachent directement à la tradition de la poésie canadienne du dix-neuvième siècle. Même les formes qu'il utilise, le grand livre de comptabilité familial dans lequel sont imprimés le texte original de «The Ledger» et la carte géographique de «Bruce County» en Ontario ainsi que le catalogue d'un marchand de graines de semences dans «Seed Catalogue» - comme celui que ses parents possédaient au temps de son enfance, eh bien ces formes sont d'un réalisme méticuleux, reproduisant même des documents d'époque! 
Mais il est très conscient de son choix. Il procède avec ironie, subvertit la réalité qu'il décrit en introduisant des structures métaphoriques et une distanciation inattendue.

V.I. Le retour aux sources n'est donc pas si simple qu'il apparaît à première vue. Le réel ne se laisse pas cerner facilement. Le poète Kroetsch le sait bien.

D.J.Exactement. Il essaie par exemple de retrouver le monde de son enfance en reconstituant le plan du jardin de sa mère. Il se souvient d'une plante d'origine japonaise, un pavot je crois. Cette plante vient de Tokyo, mais elle fait partie du monde de son enfance, des Prairies dans lesquelles il a grandi. On voit donc que le réel, la réalité géographique qu'on appelle communément l'Ouest ou les Prairies n'est pas simple du tout. À un moment donné le narrateur lit d'une main le roman The Double Hook (1959) de Sheila Watson qui se déroule au pied des montagnes de l'Alberta, près de la région de Cariboo en Colombie britannique, et en même temps il feuillette de l'autre main un livre de gravures du peintre japonais Hiroshige (1797-1858). Ce qui l'émerveille, c'est qu'il peut aussi bien s'identifier à un homme reproduit dans la gravure de Hiroshige, surpris par la pluie quelque part sur la route qui le mène de Tokyo à Kyoto, qu'à un personnage du roman The Double Hook. Un homme surpris par l'orage entre deux villes du Japon ou quelque part en Colombie britannique, quelle différence y a-t-il au fond? mon avis cela démontre à quel point il est simpliste d'essayer d'ériger des barrières entre les régions du Canada.

V.I. Ce qui me frappe comme lecteur québécois, quand je considère la question des littératures régionales au Canada, c'est le nombre d'auteurs canadiens qui vivent comme des nomades. Les écrivains se déplacent librement et vivent un peu partout dans leur pays, d'Halifax à Vancouver, et bien sûr aussi à l'extérieur du Canada. Earle Birney, né en Alberta, a souvent séjourné dans l'Est et on peut faire la même remarque au sujet de George Bowering. Margaret Atwood, née en Ontario, connaît bien plusieurs régions du Canada pour y avoir vécu. On pourrait également nommer Frank Davey, Patricia K. Page et combien d'autres. Les possibilités sont encore plus grandes de nos jours avec les programmes du Conseil des arts, les tournées de conférences, les écrivains invités par les universités, etc. Au Québec, jusqu'à tout récemment, lorsqu'un écrivain voyageait professionnellement, il se rendait infailliblement en Europe, en France la plupart du temps. Ce nomadisme des auteurs anglophones à l'intérieur du Canada n'a pas son équivalent au Québec. Le concept de littérature régionale n'a pas du tout la même résonance ici qu'au Canada ou en Europe.

D.J.Voilà une autre raison qui me rend sceptique face au bien-fondé d'une spécificité des littératures régionales au Canada. Nous sommes tous des écrivains canadiens-anglais. Les vraies différences ne sont pas tellement 
liées à la géographie ou à l'identité régionale. Elles sont plutôt culturelles. De ce point de vue la différence entre la littérature canadienne et la littérature québécoise est réelle, marquée, évidente même. Earle Birney peut aller vivre et écrire en Amérique du Sud ou en Inde, il demeure un écrivain canadien-anglais. De même Robert Kroetsch, qui a vécu des années aux États-Unis, écrit maintenant à Winnipeg, mais il est rattaché aux mêmes thèmes, à la même problématique qu'un auteur vivant en Ontario.

\section{Littératures québécoise et canadienne comparées}

V.I. C'est à partir du milieu des années soixante que vous avez commencé à donner un cours sur les littératures québécoise et canadienne comparées à l'Université de Sherbrooke. Quel impact la découverte et la lecture des écrivains québécois a-t-elle eu sur votre vision de la littérature canadienne?

D.J.Etant moi-même un poète, j'ai d'abord été attiré par la poésie québécoise, cette poésie qui était liée au mouvement moderne en Europe, depuis Mallarmé jusqu'aux contemporains. Cette conception d'une poésie symboliste et plus formelle m'a tout de suite intéressé. Je veux dire par «formelle» une poésie qui met l'accent sur la forme, les jeux avec les mots et les sons, une écriture axée sur des problèmes de langage. La littérature canadienne-anglaise mise, ou du moins semble miser, plus sur la référence au monde extérieur et par conséquent est moins attentive à la dimension formelle de la poésie, au jeu des sons et des images.

V.I. Est-ce que cet aspect de la poésie québécoise vous a surpris?

D.J.Je savais que la poésie française moderne évoluait dans ce sens. Je ne peux pas dire que cela m'a terriblement surpris de retrouver ces préoccupations formelles en poésie québécoise. Mais mon intérêt pour les poètes québécois n'en a été que plus grand. De plus, au fur et à mesure que je lisais cette poésie, je me suis rendu compte que les caractéristiques qui semblaient constituer "naturellement" la poésie canadienne n'étaient pas naturelles ou immuables du tout. Ce que Lampman, Carman et d'autres poètes écrivaient n'était pas déterminé par le fait qu'ils vivaient dans ce pays, je veux dire les descriptions d'un paysage soidisant «canadien » par exemple. Je lisais les textes de Nelligan et je m'apercevais qu'il se fichait pas mal du paysage ou de la nature. En réalité beaucoup de poètes québécois, que ce soit Crémazie ou SaintDenys Garneau dans une certaine mesure, ne s'intéressaient pas à la nature. Des auteurs francophones et anglophones, écrivant à peu près à la même époque et dans une aire géographique commune, quelquefois dans la même ville, n'avaient pas du tout la même vision du monde. J'ai da en arriver à la conclusion suivante: le sujet sur lequel Lampman écrivait et la manière dont il le faisait étaient des choix culturels.

V.I. Et ne pourrait-on pas ajouter: des choix souvent inconscients? 
D.J.On a en effet tendance, lorsqu'on est jeune, à vouloir tout simplifier: Lampman étant un poète de la fin du dix-neuvième siècle, il allait de soi puisqu'il vivait au Canada, qu'il écrivît cette poésie de la nature. Mais non, on se rend compte plus tard que l'œuvre de Lampman est un produit de la culture canadienne-anglaise. En fait le réel pour un écrivain est une interprétation culturelle. C'est une production de l'imaginaire qui ne relève pas de la réalité extérieure comme un amoncellement de pierres, une forêt ou des maisons. L'écrivain choisit continuellement, met l'accent sur ceci plutôt que cela, crée son propre monde avec son ordre et ses lois. Ces choix et cette création sont le fruit de l'imagination humaine et ils s'appuient sur une société, sur une culture.

Par exemple, même si la culture canadienne et la culture québécoise sont toutes deux des prolongements de la culture européenne, elles sont très différentes dans leur orientation et leur vision du monde. Cela ne veut pas dire qu'elles n'ont aucun point en commun: les deux cultures partagent entre autres un sentiment de solitude et d'isolement. Clément Moisan dans Poésie des frontières insiste beaucoup sur le fait qu'elles sont semblables. Eh bien elles le sont et en même temps elles ne le sont pas! Le point faible de son essai, c'est qu'il ne montre pas à quel point les deux cultures sont différentes l'une de l'autre. On peut relever un nombre important d'images de solitude et d'aliénation dans la poésie d'Anne Hébert et dans celle de Patricia K. Page, mais ce sur quoi il faut insister, ce sont sur les raisons qui les justifient. Anne Hébert se sent aliénée parce que, comme le dit François au début du conte «le Torrent», "j'étais un enfant dépossédé du monde», c'est-à-dire que le monde extérieur n'existe pas pour le narrateur qui vit replié sur son moi intérieur. Dans le cas de Patricia K. Page, c'est le phénomène contraire qui se produit: il n'y a dans son univers poétique que la réalité extérieure qui existe et la narratrice n'a pas de vie intérieure.

V.I. Une étude comparée des deux poésies devrait donc insister autant sur les oppositions que sur les similitudes entre les deux littératures et les deux cultures.

D.J.Précisément. Et une fois ces oppositions connues, si on veut pousser mon raisonnement un peu plus loin et tenter de corriger la situation, voici ce que je proposerais. Je dirais aux écrivains canadiens : apprenez à enrichir votre imaginaire, ne craignez pas d'approfondir l'univers intérieur, et aux écrivains québécois: étendez-vous dans l'espace, déployez vos antennes sur le monde extérieur. C'est d'ailleurs ce qui s'est produit avec la révolution tranquille. Le Québec, plutôt que de se satisfaire d'être quelque part et nulle part entre ici et l'éternité, a décidé de prendre sa place dans un espace concret, défini. Les écrivains sont passés à l'action et se sont approprié un lieu - Chamberland dirait qu'ils ont fondé un territoire, la terre Québec - et ils ont aussi investi le corps, le réel. 
La culture canadienne a tenté de son côté d'aller dans la direction opposée. Northrop Frye par exemple prend possession du monde de l'imagination, ce qu'il appelle l'archétype de l'imaginaire. Bien sûr cela ne se fait pas du jour au lendemain et il y a des forces de résistance tenaces. Le Canada anglais est une puissance économique, à l'instar des États-Unis; il s'est imposé grâce au commerce des fourrures, à l'industrie du bois, à l'exploitation des ressources naturelles. Or cette vision impérialiste n'a jamais été défendue par la majorité des écrivains canadiens, ou alors on y faisait référence sur le mode satirique, sauf peut-être dans le cas du poète Pratt. Le porte-parole le plus important de la culture canadienne était traditionnellement le pionnier,l'homme de la terre. Mais on sait aujourd'hui, en termes de pourcentage de la population, que l'agriculture n'existe à peu près plus dans notre société industrialisée. On voit comment la vision pastorale, dominante en littérature, s'est longtemps opposée et s'oppose encore à la vision impérialiste, dominante dans l'économie, le commerce, l'industrie.

V.I. Est-ce la raison pour laquelle on peut percevoir chez les écrivains canadiens une méfiance et même une opposition face au monde technologique moderne, face à l'arsenal nucléaire américain par exemple et à son déploiement dans le monde? La prise de conscience et l'engagement de plusieurs écrivains canadiens par rapport à ces problèmes me semblent plus importants que chez les écrivains québécois. Comment expliquez-vous cela?

D.J.La problématique centrale de la littérature canadienne repose précisément sur ce thème. Tout le Canada et non seulement les milieux littéraires ou cultivés, est divisé sur cette question: est-ce que le pays doit devenir une société libérale moderne et technologique, comme les États-Unis, ou doit-il refuser cette évolution en glorifiant sa tradition rurale qui véhicule précisément des valeurs contraires à celles de la révolution industrielle?

V.I. Comment avez-vous perçu, vers 1965-1970, le projet nationaliste québécois? Vous êtes né et avez été éduqué en Ontario mais vous résidiez au Québec pendant la Révolution tranquille. Quelle a été votre réaction à la lecture des texte littéraires nationalistes de cette époque?

D.J.Ma réaction a surtout été positive. Il faut se replacer dans le contexte de l'époque : il y avait tellement d'enthousiasme et d'énergie investis dans le projet nationaliste. Les lancements de livres, les spectacles de chansons et de poésie, les numéros de revues se suivaient à un rythme accéléré. Ce débordement d'activités, de productions littéraires et artistiques sans précédent au Québec me fascinait. C'était excitant de sentir toute cette énergie autour de soi. Mais je me sentais en même temps dans une situation ambiguë comme citoyen canadien. Fallait-il prendre parti pour un Québec indépendant? Fallait-il voter pour conserver le 
Québec à l'intérieur du Canada? Politiquement je me trouvais dans une position délicate. À bien des points de vue j'étais d'accord avec les objectifs de la Révolution tranquille: libération à l'égard d'un culte du passé qui condamnait les écrivains à répéter les textes anciens sans jamais y ajouter du nouveau, libération face à une culture traditionnelle qui conduisait à un appauvrissement collectif, libération des Québécois exploités et colonisés. J'étais donc d'accord avec cette libération et je crois que, dans un sens, cela rapprochait les Québécois des Canadiens anglais.

V.I. Étiez-vous le seul écrivain québécois de langue anglaise à penser de cette façon?

D.J.J'ai d'abord cru que le fait que John Glassco et Frank Scott traduisent Anne Hébert et Saint-Denys Garneau était paradoxal. Puis je me suis rendu compte que Scott traduisait certains textes parce qu'il se sentait en accord avec le mouvement de libération de la poésie québécoise. Il établissait probablement un parallèle entre cette révolution formelle et la Révolution tranquille. On sait le combat qu'il a mené contre le régime duplessiste au cours des années cinquante. Des écrivains comme Scott, Glassco et moi étions très impressionnés d'abord par le talent de poètes comme Saint-Denys Garneau et Anne Hébert qui parvenaient à décrire un état d'âme sans doute ressenti par des Canadiens mais peu exprimé en littérature canadienne-anglaise. Puis nous étions enthousiasmés par le fait que, une fois cet état d'âme reconnu au grand jour, les écrivains québécois pourraient dépasser ces sentiments de solitude et de désespoir, de "grande noirceur», assumer leur passé et aller de l'avant, déboucher sur «la place du monde», pour reprendre l'expression d'Anne Hébert dans Mystère de la parole. Pour Scott, cela voulait dire que le Québec devenait une des provinces «progressistes» de la social-démocratie canadienne. Quant à moi, je demeure toujours indécis quant au projet politique: le fédéralisme ou l'indépendance? Mais pendant dix ou quinze ans il était assez extraordinaire de vivre au Québec, même si nous n'étions pas nous-mêmes directement engagés dans la Révolution tranquille.

\section{La «mission» du poète-traducteur}

V.I. David Homel dans un article sur la poésie de langue anglaise au Québec vous appelle, John Glassco, Frank Scott et vous, des poètestraducteurs, un type de poète canadien-anglais particulier au Québec. Qu'est-ce qui vous a amené à la traduction?

D.J.D'abord un intérêt personnel. J'aimais la poésie française. Avec le peu de français appris au niveau secondaire, je me suis mis à traduire des poèmes ligne par ligne, mot à mot. Puis quand je suis venu à Sherbrooke au début des années soixante et que j'ai commencé à lire 
systématiquement les poètes québécois, j'ai pris conscience de la barrière linguistique qui existait entre le Québec et le Canada anglais. D'un côté comme de l'autre, on ne connaissait vraiment pas la littérature de son voisin. L'idée de créer une revue de traduction m'est alors venue car, sauf pour certains textes de Nelligan, Anne Hébert, Saint-Denys Garneau et Grandbois, la poésie québécoise était inaccessible au lecteur unilingue anglophone.

V.I. Dans un colloque sur la traduction il y a quelques années, le critique Gilles Marcotte a affirmé que Frank Scott et d'autres anglophones du Québec ont senti qu'ils avaient comme mission - c'est le mot qu'il a employé - de traduire les écrivains québécois. Que pensez-vous de cette mission?

D.J.Peut-être faut-il entendre le mot dans le sens d'une certaine solidarité avec les poèmes québécois, d'un soutien moral et d'un accord avec leur pensée, comme je l'ai expliqué tout à l'heure. Il faut se rappeler que la représentation qu'on se faisait du Québec au Canada anglais à l'époque de la Révolution tranquille était en partie folklorique, en partie idéalisée. La question qu'on retrouvait un peu partout dans les journaux, "What does Quebec want? », était due à une ignorance profonde de la nature, de la complexité de la société et de la culture québécoises. Par le biais de ses traductions, un Frank Scott par exemple peut fournir au moins une partie de la réponse : voilà ce qu'est le Québec, et ce qu'il veut est implicite si vous comprenez ce qu'il est. En fait ce qu'il veut est une toute autre affaire. Et puis il y a aussi la volonté de la part de Scott, étant donné qu'il vit au Québec, de manifester - dans le sens de rendre visibles et explicites - les deux côtés de la médaille de la société québécoise, ses deux grandes.communautés linguistiques. Ainsi les anglophones et les francophones peuvent enfin se connaître et se reconnaître, se renvoyer une image plus fidèle de l'autre.

V.I. Frank Scott l'avocat, le constitutionnaliste, le penseur socialdémocrate s'est toujours intéressé à ce qui se passait au Québec, avant, pendant et après la Révolution tranquille. Ses traductions des poètes québécois remontent à la fin des années cinquante.

D.J.Oui, Scott a toujours été un observateur de la scène politique québécoise, et plus qu'un observateur, un homme qui a pris part aux grands débats. Comme poète, il se tenait au courant de ce qui s'écrivait et de ce qui se faisait dans les milieux littéraires de Montréal, aussi bien en français qu'en anglais. À plusieurs reprises, il a souhaité un regroupement des écrivains montréalais anglophones. Il a été à sa façon un animateur littéraire avant Irving Layton et Louis Dudek, tout comme Gaston Miron l'a été pour les poètes francophones dans les années cinquante et soixante. Il a même tenté de réunir chez lui des poètes francophones et anglophones, à l'occasion de soirées qu'il animait et où il invitait les 
deux groupes à se rencontrer. Je sais que cela n'a pas vraiment fonctionné. Les canaux de communication entre les deux groupes n'étaient pas bien établis, peut-être aussi que les sujets d'intérêt commun n'étaient pas très nombreux et de toute façon il n'y avait aucun précédent, aucune tradition sur laquelle on pouvait s'appuyer pour promouvoir ces relations.

V.I. Je reviens donc à ma question originale: pourquoi Scott s'est-il donné comme mission de traduire les poètes québécois et de promouvoir les rencontres entre les deux groupes de poètes montréalais?

D.J.Je crois qu'il voulait encourager un mouvement de poésie qu'il considérait comme libérateur, mouvement commun selon lui aux poètes francophones et anglophones des années cinquante. Les deux groupes défendaient les mêmes positions, voulaient se libérer d'un sentiment de solitude et d'oppression et même de manipulation politique que Scott associait au régime de Duplessis. Les deux groupes de poètes tentaient chacun de son côté d'identifier le malaise, de décrire l'atmosphère suffocante et de s'en libérer. On voit très bien, si on se souvient des poètes que Scott traduisait - Hénault, Trottier, Giguère, Pilon -, qu'il s'agit de textes ironiques et satiriques, qui rejettent l'immobilisme de la société québécoise de l'époque. Cela sous-entend que les poètes sont à la recherche d'un modèle de société plus authentique, plus ouverte, plus libre. Cette quête coïncide avec la vision politique aussi bien que poétique de Frank Scott. Il n'est donc pas surprenant qu'il ait choisi de traduire certains poètes, certains textes québécois plutôt que d'autres.

\section{L'avenir de la traduction littéraire}

V.I. Maintenant que la traduction littéraire a pris son élan — depuis 1965 le nombre total de livres traduits du français à l'anglais et vice-versa double tous les cinq ans et même ces dernières années, dans le domaine de l'essai, il se fait plus de traductions de l'anglais au français -, croyezvous que l'époque de la "mission de traduire» est révolue? Croyezvous qu'il se fera toujours de plus en plus de traductions entre le Québec et le Canada?

D.J.Je le crois en effet, et pour toutes sortes de raisons. Les livres traduits ne sont pas tous du même niveau ou de la même qualité, c'est entendu. La traduction est devenue une industrie et il est inévitable qu'on retrouve de très bonnes mais aussi de très mauvaises traductions sur le marché, et les mauvaises ne viennent pas toujours d'où l'on pense. Les pires traductions de certains livres de Leonard Cohen par exemple nous viennent de France. On traduit pour des raisons scolaires, pour des raisons culturelles ou commerciales, pour des raisons d'avancement personnel et il y a de plus en plus de fonds provenant du Conseil des arts et d'autres organismes privés et publics pour le faire. Malgré cela la traduction littéraire est toujours sous-payée par rapport à la traduction professionnelle, gouvernementale ou scientifique. 
Au Québec, après l'élection du Parti québécois en 1976, on a senti un changement d'attitude qui, selon moi, est capital. Beaucoup d'écrivains, une fois qu'ils ont su qu'il y avait un parti politique officiellement au pouvoir pour défendre l'idée d'indépendance, n'avaient plus de raison de refuser globalement la culture du Canada anglais. Il me semble très négatif et très frustrant de voir un auteur, qui écrit des livres pour qu'ils soient vendus et lus par le plus grand nombre de gens possible, refuser de participer à des récitals ou à des rencontres à Toronto ou à Vancouver à cause d'un différend politique entre le Québec et le Canada. Cette situation s'est répétée à maintes reprises au cours de la décennie soixante-dix. Aujourd'hui les écrivains peuvent accepter les invitations de se rendre au Canada anglais sans avoir l'impression de se compromettre politiquement. Cette nouvelle attitude permet aux auteurs québécois de s'ouvrir à la littérature canadienneanglaise, de la considérer comme une entité autonome au même titre que la littérature américaine, britannique, sud-américaine...

V.I. Ce changement d'attitude est-il dû uniquement à la situation politique?

D.J.Le développement rapide des moyen de communication - télévision, radio, cinéma, vidéo-cassettes, etc. - ainsi que l'impact des États-Unis et de la culture moderne ont aussi eu des répercussions importantes sur le Québec. En ce sens la traduction au Québec, comme un peu partout dans le monde, est devenue une pratique quotidienne normale, essentielle même.

\section{La littérature québécoise de langue anglaise}

V.I. Nous avons parlé de ce paradoxe apparent de l'écrivain québécois qui, depuis la prise du pouvoir par le Parti québécois, a changé d'attitude et est plus ouvert que jamais à la littérature et à la culture canadiennes. Ce qui semble encore plus paradoxal, c'est que la littérature québécoise de langue anglaise, qui se trouvait dans un état de léthargie depuis le milieu des années soixante, s'est mise à donner des signes de vie précisément à la suite de la conquête du pouvoir par le $\mathrm{PQ}$. Je pense à des poètes comme David Solway et Michael Harris ou à ceux du groupe montréalais «Véhicule» très actifs de 1977 à 1982 (Endre Farkas, Artie Gold, Tom Konyves, Claudia Lapp, John McAuley, Stephen Morrissey, Ken Norris), à la quinzaine de petites maisons d'édition littéraire rassemblées dans le catalogue publicitaire de Véhicule, aux anthologies Cross/cut. Contemporay English Quebec Poetry et The Insecurity of Art. Essays on Poetics publiées ces dernières années. Comment expliquer ce paradoxe: la renaissance de la poésie québécoise de langue anglaise à Montréal qui survient, comme par hasard, tout de suite après l'élection d'un parti qui vote la loi 101 et met l'accent sur la défense des intérêts des francophones au Québec? 
D.J.Je ne suis pas sûr de pouvoir expliquer ce paradoxe de façon tout à fait satisfaisante. Une chose est certaine: vers la fin des années soixante les poètes aînés, ceux qui étaient les porte-parole de la poésie de langue anglaise au Québec, ont soit presque cessé d'écrire et de publier, soit quitté Montréal et le Québec. C'est le cas de Irving Layton, de Leonard Cohen, de Louis Dudek, de Frank Scott. La plaque tournante de la poésie canadienne-anglaise s'est déplacée de Montréal vers Toronto et Vancouver. Il y a eu un temps mort, une période d'une dizaine d'années pendant laquelle il ne s'est pas publié beaucoup de livres. Le poète et éditeur Louis Dudek se trouvait toujours à McGill, mais devant les nouvelles tendances en poésie canadienne et à la suite du mouvement général vers Toronto et Vancouver, il était nettement moins enthousiaste et moins entreprenant.

\section{V.I. Alors qu'est-ce qui s'est passé pour que ça démarre de nouveau?}

D.J.Il a fallu attendre qu'une nouvelle génération de poètes voie le jour. Ces poètes d'ailleurs viennent d'un peu partout à travers le monde, d'Italie, des États-Unis, d'Europe de l'Ouest et de l'Est, et aussi de différents milieux sociaux. Frank Scott, Arthur Smith et même Abraham Klein constituaient, dans la période des années trente et quarante, un groupe relativement homogène de poètes formés à l'université (McGill en particulier). Influencés par la littérature canadienne traditionnelle, ils en étaient le dernier chaînon. Les jeunes poètes des années quatre-vingt comme Antonio d'Alfonso, Ken Norris ou Peter Van Toorn viennent d'autres pays, prônent de nouvelles conceptions de la poésie, en somme ne s'appuient pas sur cet élément de continuité de la littérature canadienne. Ces poètes créent donc un nouveau mouvement, fondent des maisons d'édition, écrivent une poésie qui marque une rupture par rapport à la poésie de langue anglaise au Québec. "Véhicule» comptait des dizaines de poètes à un moment donné; c'est une époque d'effervescence tout à fait saine car, de ce groupe d'écrivains, certains continueront à écrire et à publier alors que d'autres disparaîtront.

V.I. Peut-on considérer que c'est la situation politique du Québec qui a servi de bougie d'allumage a cette nouvelle génération de poètes? Vers 1977-1978 n'était-il pas téméraire et même un peu suspect d'écrire en anglais au Québec? Il devait certainement y avoir de la part des poètes anglophones un certain sens du défi, de l'aventure à se regrouper et à créer un mouvement de poésie de langue anglaise à Montréal.

D.J.Je ne crois pas que ces jeunes poètes soient politiquement engagés. Ils sont peut-être plus engagés dans le sens romantique du mot et d'une certaine façon désillusionnés sur le plan politique. Ce qu'ils publient ne ressemble pas à ce que publiaient les poètes francophones du Québec des années soixante et soixante-dix. Ces derniers ont du reste pris leurs 
distances par rapport à la politique. En fait les deux mouvements de jeune poésie au Québec, francophone et anglophone, se tournent vers l'avenir et explorent de nouvelles voies. Il y a longtemps que j'espérais cette bouffée d'air frais, cette liberté dans l'expérimentation. Toutes les possibilités sont là ainsi que la volonté des jeunes poètes d'aller de l'avant. Il n'y a qu'à se lancer dans de nouvelles directions. Je suis avec intérêt par exemple la démarche de Michel Beaulieu qui se rend sur la côte du Pacifique depuis quelques années, rencontre les poètes de la Colombie britannique, lit ce qui se fait de nouveau, s'en imprègne et revient ici pour les traduire et les faire connaître. C'est ce que j'appelle être ouvert à ce qui se fait au Canada anglais. L'attitude de Beaulieu n'est pas agressive, défensive ou satirique, mais sympathique. Je crois que cette nouvelle attitude est tout à fait bénéfique pour les deux groupes de poètes. 